\title{
Philonsorbonne
}

11 | 2017

Année 2016-2017

\section{Diderot et la légitimation philosophique de la révolution}

\section{Kyosuke TAHARA}

\section{OpenEdition}

1 Journals

Édition électronique

URL : https://journals.openedition.org/philonsorbonne/867

DOI : $10.4000 /$ philonsorbonne.867

ISSN : 2270-7336

Éditeur

Publications de la Sorbonne

Édition imprimée

Date de publication : 1 janvier 2017

Pagination : 53-73

ISSN : 1255-183X

\section{Référence électronique}

Kyosuke TAHARA, «Diderot et la légitimation philosophique de la révolution », Philonsorbonne [En ligne], 11 | 2017, mis en ligne le 07 janvier 2017, consulté le 08 juin 2021. URL : http:// journals.openedition.org/philonsorbonne/867; DOI : https://doi.org/10.4000/philonsorbonne.867

(c) Tous droits réservés 


\title{
Diderot et la légitimation philosophique de la révolution
}

\author{
Kyosuke TAHARA
}

\section{Diderot contre le contractualisme hobbesien}

Le discours apologétique de la Révolution américaine de 1776 que Diderot écrivit pour l'insérer anonymement dans la troisième édition de l'Histoire des deux Indes de l'abbé Raynal (1780) ${ }^{1}$ constitue assurément un texte non négligeable pour le lecteur qui aurait l'intention de poser un jalon attestant la radicalisation de sa pensée politique. Mais rien ne serait plus hâtif que de déduire de cette apologie que Diderot aurait considéré la révolution comme positive en toutes circonstances. L'ambivalence semble tout à fait incontestable. Si l'on se réfère aux Mélanges philosophiques, historiques, etc., reconfigurant ses entretiens avec Catherine II lors de son voyage en Russie d'octobre 1773 jusqu'en mars 1774, on y voit en effet un philosophe qui prêche des mesures préventives contre les révolutions que pourraient entreprendre des seigneurs appauvris, tout en insérant une

\footnotetext{
1. Pour citer la collaboration du philosophe à l'ouvrage de Guillaume Thomas Raynal, je renverrai à la fois à : Histoire philosophique et politique des établissements et du commerce des Européens dans les deux Indes, Genève, chez J.-L. Pellet, 1780, 4 vol. in-4º (désormais HDI) ; et à : Denis Diderot, Pensées détachées. Contributions à l'Histoire des deux Indes, t. I, Gianluigi Goggi (éd.), Siena, 1976 (désormais PD); Mélanges et morceaux divers. Contributions à l'Histoire des deux Indes, t. II, Id. (éd.), Siena, 1977 (désormais Mél). Voir généralement Michèle Duchet, Diderot et l'Histoire des deux Indes ou l'Écriture fragmentaire, Paris, Nizet, 1978. Voir aussi un article de Goggi, qui est en charge de l'édition de cette collaboration qui sera insérée dans les Euvres complètes de Diderot en cours de publication chez Hermann: "La collaboration de Diderot à l'Histoire de deux Indes : l'édition de ses contributions », Diderot Studies, XXXIII, Genève, Droz, 2013, p. 167-212.
} 
suggestion qui semble autoriser les révolutions populaires ${ }^{2}$. De même, dans la deuxième édition de l'Essai sur Sénèque parue sous le titre d'Essai sur les règnes de Claude et de Néron deux ans avant sa mort en 1784, alors que l'éloge de la Révolution américaine demeure intact, Diderot défend Sénèque qui prit des précautions pour éviter une révolte populaire contre la tyrannie de Néron, dans la mesure où le malheur des Romains aurait été « très grand, si la révolution ne pouvait guère s'exécuter qu'en faisant couler des flots de sang ${ }^{3}$. Cependant, il n'est pas nécessaire de diagnostiquer ici une contradiction, d'autant que le discours politique du dernier Diderot est loin de se déduire d'une spéculation préétablie ; comme l'a observé avec justesse G. Dulac, il dispose « de plusieurs modes d'interventions différents, adaptés aux visées pratiques » selon les circonstances, qu'il s'agisse de déclarer la vérité générale sur le bien public, d'appeler avec indignation l'agitation politique contre l'injustice, ou de juger des tactiques destinées à infléchir une situation vers une issue plus ou moins heureuse ${ }^{4}$.

Malgré cette importance accordée aux circonstances, peut-on reconstituer l'idée de révolution chez Diderot et mesurer sa portée philosophique ? Une piste se trouve peut-être dans le fait qu'il commence sa justification de la Révolution américaine en remontant jusqu'à l'origine de la société, pour autant que ce geste suggère qu'il tente de penser la consistance d'une politique légitime en concurrence avec la théorie politique de Hobbes. On sait que le contractualisme hobbesien a été élaboré dans la crainte du déferlement de la guerre civile qui faisait partie de l'arrière-plan politique de l'Angleterre du dix-septième siècle ${ }^{5}$. Les efforts de Hobbes ont tendu à légitimer théoriquement l'État pourvu d'un pouvoir souverain, en esquissant sa genèse hypothétique à partir de l'état de nature assimilé à une "guerre de chacun contre chacun», et en refusant farouchement toute sédition susceptible de provoquer un retour effectif à cet état anarchique ${ }^{6}$. En un mot, dans le système hobbesien, toute tentative de révolution politique se trouve condamnée.

2. Mélanges philosophiques, historiques, etc., pour Catherine II, in Euvres, t. III, Laurent Versini (éd.), Paris, Robert Laffont, 1995, désormais MC2 - ici, p. 348 : «Il [le philosophe] dit aux peuples qu'ils sont les plus forts et que, s'ils vont à la boucherie, c'est qu'ils s'y laissent mener. Il prépare aux révolutions, qui surviennent toujours à l'extrémité du malheur, des suites qui compensent le sang répandu ».

3. Essai sur les règnes de Claude et de Néron, in Euvres complètes, Herbert Dickermann, Jacques Proust et Jean Varloot (éds.), Paris, Hermann, 2004, t. XXIV, p. 144. En suivant l'usage, nos références à cette édition seront désignées DPV.

4. Georges Dulac, «Les modes d'interventions de Diderot en politique », in Diderot. Les dernières années, 1770-1784, Peter France et Anthony Strugnell (éds.), Edimbourg, Edimbourg University Press, 1985, p. 121-139.

5. Sur la théorie absolutiste de Hobbes et son rapport à l'histoire, cf. Reinhart Koselleck, $L e$ Règne de la critique, trad. Hans Hildenbrand, Paris, Minuit, 1979, p. 19-33.

6. Thomas Hobbes, Du citoyen, trad. Philippe Crignon, Paris, Flammarion, 2010, «Préface aux lecteurs », p. 85 : «lorsqu'on recherche le droit de l'État et les devoirs [officiis] des citoyens, il est nécessaire non pas, certes, de dissoudre l'État, mais néanmoins de le considérer comme dissous [dissoluta]». Voir aussi Léviathan, trad. François Tricaud, Paris, Sirey, 1971, ch. 28, p. 338 : «la rébellion n'est que la reprise de l'état de guerre ». 
Face à la guerre d'indépendance de la colonie de l'Amérique septentrionale à l'encontre de l'Empire britannique, Diderot a pris clairement conscience qu'il y avait un contraste entre cette nouvelle réalité politicohistorique et la doctrine philosophique qui semble la nier: "Nous examinons les choses en philosophes; et l'on sait bien que ce ne sont pas nos spéculations qui amènent les troubles civils. Point de sujets plus patients que nous. Je vais donc suivre mon objet, sans en redouter les suites ${ }^{7}$. Manifestement, il ne pouvait pas s'empêcher d'être fidèle à cet événement révolutionnaire, tout en adoptant la posture du philosophe. Une telle position l'a conduit sans doute à combler l'écart entre l'objet inédit et la théorie invalidée ; c'est-à-dire à justifier ladite réalité, ce qui était injustifiable dans la philosophie politique de Hobbes, selon laquelle on était pris dans l'alternative suivante: obéissance inconditionnelle au souverain absolu ou rébellion légitimement destinée à la répression. Toutefois, pour établir le bien-fondé d'une révolution, ce n'est pas assez de prendre le contre-pied de l'absolutisme hobbesien, en revendiquant tout simplement le droit de s'insurger contre le pouvoir répressif de l'État. Il faut encore renouveler la méthode ${ }^{8}$ de légitimation qui met également en jeu l'historicité de l'institution civile. En effet, quoique Diderot remonte vers son origine, cette régression n'a plus pour but de renvoyer ce qui est légitime au schème abstrait du pacte social, mais d'introduire un autre schème qui est celui de la civilisation, visant à dessiner l'histoire de la société civile sans y mêler le moment contractuel ${ }^{9}$. Si le philosophe s'applique à justifier la révolution dans une temporalité qui ne laisse aucune place au contrat fictif, c'est que, comme chaque situation concrète exige sa propre solution pratique, il ne s'agit pas de déduire ce qu'il convient de faire de ce qui s'est révélé préalablement légitime par l'argumentation abstraite; il s'agit plutôt de reconstituer le processus général, appelé civilisation, qui pourrait s'appliquer aux diverses circonstances parmi lesquelles figure la Révolution américaine, ainsi que de savoir dans quelle condition historique la Révolution d'indépendance telle qu'elle s'est effectivement déroulée dans le Nouveau Monde était

7. HDI, t. IV, liv. 18, ch. 42, p. 393 ; Mél, p. 151 (souligné par nous).

8. Je m'inspire ici librement de l'orientation de la lecture que Louis Althusser a proposée de Montesquieu (Montesquieu, la politique et l'histoire, Paris, Presses Universitaires de France, 1959, p. 15).

9. Sur la confrontation de ces deux schèmes dans la philosophie des Lumières, on peut consulter la bonne synthèse de Bertrand Binoche, «Échange, contrat et civilisation », Revue de Synthèse, t. $129,6^{\mathrm{e}}$ série, $\mathrm{n}^{\circ} 1,2008$, p. 85-103. L'emploi du schème de la civilisation chez Diderot et son attention aux circonstances empiriques indiquent assurément sa dette non négligeable envers Montesquieu, et à cet égard il me semble nécessaire de compléter le travail brillant, mais partiel, de Colas Duflo qui a mis à jour certains aspects de l'application par Diderot de la pensée de Montesquieu à l'interprétation de la réalité politique de son temps autour de l'affaire de Maupeou («Peut-on lire en philosophe sa propre actualité politique ? Le dernier Diderot et l'héritage de Montesquieu », Diderot Studies, XXXII, Genève, Droz, 2012, p. 25-45). 
justifiable, voire réalisable. Ce qui est en cause, c'est, si l'on peut dire, un mode d'institution circonstancielle de facto de ce qui est de jure.

De la sorte, la réflexion de Diderot sur la révolution a pour enjeu à la fois de renverser axiologiquement l'indissolubilité du pouvoir étatique, et d'inventer un nouveau dispositif de justification théorique sous la forme de la temporalité de la civilisation. C'est en suivant cette double confrontation avec le contractualisme hobbesien que nous voudrions examiner le discours diderotien de la Révolution américaine et ses derniers écrits politiques relatifs à celle-ci, afin d'en dégager l'idée de révolution en la rapportant aux éléments conceptuels concernés (l'origine de la société, l'inégalité, la civilisation, la souveraineté, etc.). Cependant, pour mesurer plus précisément sa portée philosophique, nous évoquerons également la confrontation du philosophe avec d'autres positions significatives (Hume, Rousseau et Locke notamment).

\section{L'origine double de la société et du gouvernement}

Commençons par l'interprétation diderotienne du point de départ de la civilisation. Elle entraîne d'emblée un refoulement de l'état de nature et une séparation de la société tout court et du gouvernement ${ }^{10}$. Pour un contractualiste tel que Locke, une société digne de ce nom n'est pas autre chose que la «société civile ou politique » qui possède une autorité commune, de telle sorte qu'un rassemblement des hommes sans convention pour mettre en place ce dispositif, s'il a lieu, relève encore de l'état de nature $^{11}$. A contrario, «il faut bien, déclare Diderot dans l'Histoire des deux Indes, se donner de garde de confondre ensemble les sociétés et les gouvernements $»^{12}$, étant donné le décalage temporel entre les deux : la société se forme naturellement sans que cette formation soit sectionnée par une discontinuité conventionnelle, et le gouvernement se greffe graduellement sur elle. En ce sens, on peut et doit se passer de la temporalité du contractualisme ainsi que de l'état de nature statique qui fait apparaître

10. Il est tout à fait légitime de relever ici le langage d'un autre défenseur de la cause de la Révolution américaine, Thomas Paine qui a distingué au début du Sens commun (1776) la société comme bien originel et du gouvernement comme mal nécessaire (Common Sense, in Political Writings, Bruce Kuklick (éd.), Cambridge, Cambridge University Press, 1989, p. 3-5), d'autant plus que Diderot cite son nom. Mais, si l'on se réfère à la Suite de l'Apologie de M. l'abbé de Prades (1752) dans laquelle Diderot avait ordonné ses idées parsemées des articles de l'Encyclopédie au sujet de l'origine de la société, on peut remarquer aisément qu'il avait déjà séparé l' «état de troupeau » ou la société naturelle et la « société policée » pourvue d'un gouvernement (DPV, t. IV, p. 334 ; p. 349).

11. John Locke, Le second Traité du gouvernement, trad. Jean-Fabien Spitz et Cristian Lazzeri, Paris, Presses Universitaires de France, 1994, § 15, p. 145 : « J'affirme, en outre, que tous les hommes sont naturellement dans cet état [de nature] et qu'ils le restent jusqu'à ce que, de leur consentement, ils deviennent membres d'une société politique ».

12. HDI, t. IV, liv. 18, ch. 42, p. 391 ; Mél, p. 148. 
toute institution sociale et politique instantanément et ex nihilo à travers la convention : à preuve ce que Diderot dit explicitement dans les Observations sur le Nakaz: "Les hommes se sont réunis en société par instinct, comme les animaux faibles se mettent en troupeau. Il n'y a certainement eu primitivement aucune sorte de convention ${ }^{13}$.

De la naturalité de la collectivité sociale, on peut conclure qu'il est hors de question d'argumenter sur l'existence réelle d'un état asocial. C'est pourquoi Diderot n'a rien d'autre à faire que de « rêver ${ }^{14}$ hypothétiquement un passage de l'état antérieur à la société. Or, cette régression conjecturale n'est-elle pas analogue à la manière dont Rousseau a prétendu, dans le Discours sur l'origine et les fondements de l'inégalité parmi les hommes, écarter «tous les faits » et s'en rapporter à «des raisonnements hypothétiques et conditionnels » pour supposer l'état fictif et révélateur de la nature humaine ${ }^{15}$ ? En réalité, et de toute évidence, leurs intentions s'opposent: tandis que la conjecture rousseauiste met en relief la contingence plus ou moins infortunée de la sortie de l'état de nature dans lequel l'homme isolé se suffisait à lui-même, la rêverie diderotienne tâche de montrer la nécessité d'une formation non contractuelle de la société qui permette à l'homme de survivre. Aussi Diderot avance-t-il une hypothèse qui fait la preuve de l'auto-organisation ${ }^{16}$ des hommes en une société tendue vers un objet commun, c'est-à-dire qu'il souligne la nécessité pour les hommes de lutter ensemble contre la nature : faute d'un abri contre «tous les maux de la nature » tels que les catastrophes naturelles, le mauvais temps, la famine, l'attaque des bêtes sauvages, etc., "l'homme, dans cet état, seul et abandonné à lui-même, ne pouvait rien pour sa conservation. Il a donc fallu qu'il se réunît et s'associât avec ses semblables, pour mettre en commun leur force et leur intelligence ${ }^{17}$.

Mais il faut prendre garde de ne pas amalgamer cette cause extérieure de la société et « un germe de sociabilité » que Diderot regarde comme « une des premières facultés de l'homme » et qui le dirige vers une « double fin de

13. Observations sur le Nakaz, in Cuvres, t. III, Laurent Versini (éd.), Paris, Robert Laffont, 1995, § 72, p. 544 (désormais Observ).

14. MC2, XXVIII, p. 312.

15. Jean-Jacques Rousseau, Discours sur l'origine et les fondements de l'inégalité parmi les hommes, in Euvres complètes, Bernard Gagnebin et Marcel Raymond (éds.), Paris, Gallimard, Bibliothèque de la Pléiade, 1964, t. III, p. 132-133. Locke affirme aussi qu'il vaut «mieux de ne pas trop explorer l'origine des gouvernements tels qu'ils ont commencé de facto », afin de maintenir la pertinence de jure de son esquisse de la formation d'une société politique (op. cit., $\S 103$, p. 75).

16. Terme emprunté à Gehardt Stenger, Nature et liberté chez Diderot après l'Encyclopédie, Paris, Universitas, 1994, p. 291-299; voir aussi Annie Ibrahim, Diderot. Un matérialisme éclectique, Paris, Vrin, 2010, p. 200-203.

17. HDI, t. IV, liv. 18, ch. 42, p. 391 ; Mél, p. 148. On peut constater la récurrence de cette même idée dans plusieurs textes de Diderot dans les années 1770, cf. par exemple Supplément au Voyage de Bougainville [1772], DPV, t. XII, p. 628 ; Fragments politiques échappés du portefeuille d'un philosophe [1772], G. Goggi (éd.), Paris, Hermann, 2011, p. 111-112; Réfutation d'Helvétius [1777], DPV, t. XXIV, p. 721. 
la nature » à savoir la «reproduction » et la «conservation », en refusant implicitement les positions de Hobbes et de Rousseau, qui ont également supposé « un état sauvage, idéal \& chimérique ${ }^{18}$. En toute rigueur, il y a une relation de potentialité à actualisation entre la sociabilité naturelle comme première faculté humaine et la rivalité avec la nature comme première condition humaine ; car précisément, comme Diderot en fait la supposition dans les Observations sur le Nakaz, si l'on ne faisait pas face à la menace de la nature, on n'en arriverait jamais à s'entraider pour la vaincre: «Faites que la nature soit une meilleure mère et que la terre satisfasse à tous les besoins de l'homme, sans en exiger aucun travail, et à l'instant vous dissoudrez la société ${ }^{19}$. De ce point de vue, on peut dire que la nécessité de lutter contre la nature est une cause primaire qui actualise immédiatement l'instinct de sociabilité en puissance et presse l'homme de former une sociétée ${ }^{20}$. Au reste, une telle cause est aussi constante dans la mesure où elle «n'a point eu de commencement et [...] n'aura point de fin ». Il s'ensuit alors que les hommes ont dû, doivent et devront toujours se réunir en société contre la nature. Dès lors, l'état social est une condition à la fois primordiale et perpétuelle pour l'espèce humaine. Cela n'exclut pas que son état varie géographiquement et en fonction du cours du temps dans lequel chaque société marche d'une allure différente. Ainsi, comme nous y reviendrons un peu plus loin, Diderot se demandera comment faire advenir effectivement ce qui est de jure à partir de conditions de facto variables une telle attitude sera sous-jacente à sa conception de la révolution comme constituant une des issues de la civilisation.

Or, lorsque le philosophe remplace l'origine contractualiste par la sienne, sa cible reste la théorie de Hobbes, à qui il donne cette fois l'assaut de l'intérieur. En effet, en attachant de l'importance à un moment agonistique donnant lieu à une solidarité parmi des intéressés contre la menace de l'extérieur, Diderot adopte certes la notion hobbesienne d'association, mais cette référence a pour objectif d'en renverser les significations. Selon Hobbes, bien que le concours ou «la convergence de plusieurs volontés » face à l'ennemi extérieur donne naissance à une association comme celle d'un troupeau d'animaux, cela ne suffit pas à prendre des mesures contre des risques de conflit entre les hommes; et par conséquent cela ne suffit pas non plus à les faire sortir de l'état de nature ; pour former la société civile, il faut que chacun soumette, par contrat réciproque, sa propre volonté à « une volonté singulière de tous » (c'est-àdire qu'il faut composer une union) et transfère tout son droit à un souverain (personne civile) qui, par son pouvoir absolu, permette aux hommes de vivre

18. HDI, t. IV, liv. 19, ch. 2, p. 470-471; PD, p. 52-53; p. 73.

19. Observ, § 71, p. 544.

20. D’après Goggi, Diderot reprend ici la conception de la sociabilité chez Spinoza pour polémiquer contre Rousseau qui fait de l'asociabilité une disposition naturelle de l'homme (De l'Encyclopédie à l'éloquence républicaine. Études sur Diderot et autour de Diderot, Paris, Champion, 2013, ch. 4 : «Spinoza contre Rousseau : un commentaire de certains passages de Diderot et de d'Holbach », notamment p. 171-174). 
pacifiquement ${ }^{21}$. Diderot s'oppose d'abord à Hobbes, en ce qu'il identifie comme ennemi extérieur non pas l'autre tribu, mais la nature - en cela il s'aligne peut-être sur une tradition du matérialisme antique, dans le style de celui de Lucrèce par exemple ${ }^{22}$. En second lieu, et cela est plus important, Diderot considère le concours de volontés sans unité ni soumission à l'Un comme une condition suffisante pour que les hommes forment spontanément une société : «Le mot de société fait concevoir un état de réunion, de paix, de concours des volontés de tous les individus vers un but commun ${ }^{23}$. Cette substitution de la société à l'association ne se borne pas au problème de la dénomination; en qualifiant de société une collectivité indépendante de toute structure de pouvoir, Diderot lui attribue aussi une signification politique que Hobbes ne lui aurait jamais reconnue : la société peut devenir le cas échéant un sujet en conflit avec son gouvernement.

La société en tant que collaboration spontanée des hommes précède donc à la fois temporellement et axiologiquement le gouvernement; et ceci a pour corollaire un rapport entre les deux de fin à moyen : «La société est la première, elle est dans son origine indépendante et libre ; le gouvernement a été institué pour elle et n'est que son instrument. C'est à l'une à commander : c'est à l'autre à la servir »; concrètement, le gouvernement a en principe pour fonction de "prévenir et de réprimer les injures que les associés avaient à craindre les uns de la part des autres $»^{24}$, afin d'assurer la préservation mutuelle des droits essentiels de chacun, tels que la liberté et la propriété. Sur ce point, Diderot se fonde certainement sur l'idée lockéenne des fins du gouvernement ${ }^{25}$. Seulement, il étend quelque peu ces fins ; car ce n'est pas tant la protection elle-même de la liberté et de la propriété qui est le plus précieux que bien plutôt ce qu'elle permet aux ayants droit, id est le bonheur des individus. À cette fin, dans les Observations sur le Nakaz, Diderot charge le gouvernement d'aménager en quelque sorte l'égalité économique qui permet à tous de jouir de l'aisance par leur activité productrice, en mettant en place par exemple l'impôt progressif et équitable et l'offre de terres et de moyens de production pour tous les paysans pauvres $^{26}$. Mais tout cela n'est qu'un moyen de se procurer le bonheur, pour autant qu'il consiste en des activités auxquelles chacun s'applique au-delà de la reproduction économique :

21. Hobbes, Du citoyen, op. cit., V, p. 160-163. Son premier traité des Éléments de la loi naturelle et politique fait la même distinction, cf. De la nature humaine, trad. Paul-Henry Dietrich d'Holbach, Paris, Vrin, 1971, p. 148-149.

22. Cf. Bertrand Binoche, «Note sur Diderot et la civilisation », in L'édition du dernier Diderot. Pour un Diderot électronique, Gianluigi Goggi et Didier Kahn (éds.), Paris, Hermann, 2007, p. 21-22.

23. Observ, § 69, p. 543.

24. HDI, t. IV, liv. 18, ch. 42, p. 391-392 ; Mél, p. 148-149.

25. Locke, op. cit., §9, p. 93 : les hommes en société confient tous leur pouvoir entre les mains du gouvernement pour qu'il «en dispose selon que le bien de cette même société l'exigera $[\ldots]$ dans l'intention de préserver d'autant mieux sa personne, sa liberté et sa propriété ».

26. Observ, § 78, p. 547 ; § 80, p. 547. 
«Si la liberté et la propriété sont assurées, ne serait-il pas permis à un citoyen d'employer sa richesse selon son goût? Pourquoi devient-on riche ? Est-ce pour être riche ? C'est pour être heureux. [...] Si l'homme n'est fait que pour labourer, recueillir, manger et vendre, tout est bon; mais il me semble qu'un être qui sent est fait pour être heureux par toutes ses pensées $»^{27}$.

Autrement dit, le gouvernement existe moins pour un homo æeconomicus que pour un homme eudémonique qui poursuit sa propre félicité à sa manière. Et sa légitimité dépendra de l'opinion des individus qui jugent eux-mêmes de ce qui est susceptible de rendre leur sort heureux.

Reste à répondre à une question supplémentaire : si la bonté naturelle de la société est indéniable, d'où provient le vice qui force la société à constituer un gouvernement ? Il semble que Diderot identifie trois obstacles qui dégradent l'intégrité de la société : les besoins dénaturés, l'inégalité naturelle et l'état de guerre. Primo, aussi longtemps que les hommes se réunissent en société en vue de leur conservation mutuelle par peur de la nature, leurs besoins sont naturellement bons; mais quand ils ne s'en tiennent plus à cette fin originaire, leurs besoins commencent à se dénaturer et à se multiplier démesurément : «Le mal est qu'ils ont passé le but. Ils ne se sont pas contentés de vaincre, ils ont voulu triompher; ils ne se sont pas contentés de terrasser l'ennemi, ils ont voulu le fouler aux pieds ; de là la multitude des besoins artificiels ${ }^{28}$. Secundo, d'autant que l'inégalité de «talent », « force », « ressources », « moyens de défense », « qualités d'esprit et de corps $\gg{ }^{29}$ existe indéniablement parmi les hommes, à peine se rassemblent-ils en société que des disputes entre les faibles et les forts ont lieu ; et par là, ils se mettent tous en péril, sauf s'ils s'obligent mutuellement à obéir aux lois et à l'autorité commune ${ }^{30}$. Dans les deux cas, soit l'inégalité, soit le besoin, les maux potentiels dans la nature ont dû s'actualiser aussitôt que l'homme a mis les pieds dans l'état social. Tertio, de ce fait, on voit clairement pourquoi Diderot situe, à l'instar de Montesquieu ${ }^{31}$, l'état de guerre après le commencement d'une société, et non point avant celui-ci comme chez Hobbes qui considère l'état de guerre comme une conséquence

\section{Observ, § 73, p. 545.}

28. Observ, §71, p. 544. Voir aussi l'article «Besoin» dans Encyclopédie, DPV, t. VI, p. 179 ; «Agriculture» dans Encyclopédie, DPV, t. V, p. 292 ; et notamment Supplément au Voyage de Bougainville, DPV, t. XII, p. 628.

29. HDI, t. IV, liv. 18, ch.42, p. 392 ; Mél, p. 148. Pour son ami le baron d'Holbach, l'inégalité naturelle n'est pas une semence latente de discorde, mais une cause immédiate de la sociabilité par laquelle chacun compense chaque défaut (Système de la nature, Josiane Boulad-Ayoub (éd.), Paris, Fayard, 1990, t. I, ch. 9, p. 150-151).

30. Cf. Suite de l'Apologie de M. l'abbé de Prades, DPV, t. IV, p. 348 : « C'est que, n'étant encore enchaînés par aucunes lois, animés tous par des passions violentes, cherchant tous à s'approprier les avantages communs de la réunion selon les talents de force, de sagacité, etc. que la nature leur a distribués en mesure inégale ; les faibles seront les victimes des plus forts ; les plus forts pourront à leur tour être surpris et immolés par les faibles ».

31. De l'esprit des lois, Robert Derathé (éd.), Paris, Garnier Frères, 1973, t. I, liv. 1, ch. 3. 
nécessaire du fait que les hommes sont naturellement égaux en ce qu'ils peuvent s'égorger ${ }^{32}$. Selon le premier, c'est en passant un seuil ou en effectuant un «saut qualitatif ${ }^{33}$ au cours du processus de socialisation (à savoir l'actualisation de l'inégalité et la dénaturation des besoins) que du corps social résulte l'état de guerre : "Mais lorsqu'en les [= les hommes comme ressorts] rassemblant et les ordonnant on en eut formé ces énormes machines qu'on appelle sociétés [...] on créa artificiellement un véritable état de guerre $»^{34}$.

\section{De l'inégalité irrémédiable à son issue révolutionnaire}

Selon les analyses qui précèdent, on peut résumer ainsi le processus que Diderot a esquissé : le conflit originaire de l'homme avec les maux de la nature nécessite la société, puis la perversion de la société nécessite le gouvernement, étant entendu que celui-ci doit servir de moyen à celle-là. Tout se passe comme si une solution nécessaire à un mal produisait un autre mal qui exigerait à son tour une autre solution. Mais il est à noter que la naissance du gouvernement est loin de clore le problème. En effet, la fondation d'une société politique enclenche la tension entre société et gouvernement dans la mesure où celui-ci «ne peut [être] et n'est que trop souvent mauvais $»^{35}$ : c'est-à-dire que la mise en place d'un gouvernement, en tant que dernier terme, annonce moins l'arrivée d'un ordre pacifique que l'inauguration véritable d'un processus fluctuant de la civilisation hantée par la corruption du gouvernement. S'agissant de tracer cet itinéraire qui mène au point où peut survenir une révolution, Diderot revient sur la contradiction originaire de l'inégalité naturelle :

«Il y a entre les hommes une inégalité originelle à laquelle rien ne peut remédier. Il faut qu'elle dure éternellement ; et tout ce qu'on peut obtenir de la meilleure législation, ce n'est pas de la détruire ; c'est d'en empêcher les abus. [...] Que les fondateurs des nations, que les législateurs se sont-ils donc proposés ? D'obvier à tous les désastres de ce germe développé, par une sorte d'égalité artificielle, qui soumît sans exception les membres d'une société à une seule autorité impartiale. C'est un glaive qui se promène indistinctement sur toutes les têtes : mais ce glaive était idéal. Il fallait une main, un être physique qui le tînt. Qu'en est-il résulté ? C'est que l'histoire de l'homme civilisé n'est que l'histoire de sa misère. Toutes les pages en sont teintes du sang, les unes du sang des oppresseurs, les autres du sang des opprimés. [...] Dans une même société, il n'y a aucune condition qui ne dévore et qui ne soit dévorée, quelles

32. Hobbes, Du citoyen, op. cit., I, p. 100-101.

33. Terme emprunté à Michèle Duchet, Anthropologie et histoire au siècle des Lumières, Paris, Albin Michel, 1995, p. 427.

34. HDI, t. IV, liv. 19, ch. 2, p. 470 ; PD, p. 52. Même idée dans MC2, XXVIII, p. 311.

35. HDI, t. IV, liv. 18, ch. 42, p. 392 ; Mél, p. 149. 
qu'aient été ou que soient les formes du gouvernement ou d'égalité artificielle qu'on ait opposées à l'inégalité primitive ou naturelle ${ }^{36}$.

Puisque les hommes entrés dans l'état social ont été forcés de contenir les conséquences fâcheuses de l'inégalité naturelle entre chacun, ils ont mis en œuvre une souveraineté susceptible de leur garantir l'égalité artificielle par sa législation et son pouvoir exécutif. Mais le fait que la personne morale qu'est la souveraineté ait dû être incarnée dans une certaine personne physique est devenu la véritable pierre d'achoppement pour les hommes civilisés. Car même si l'on estime, comme chez Hobbes, que l'épée souveraine aurait dû être mise en œuvre de jure par un homme ou par un assemblée ${ }^{37}$, aux yeux de Diderot, elle a toujours sévi de facto avec intempérance. En outre, le plus souvent, le rapport de fin à moyen entre société et gouvernement a été renversé de telle façon que «toutes les sociétés n'ont pour principe ou pour suprême loi, que la sûreté de la puissance dominante ${ }^{38}$. Et en conséquence, «par sa nature, toute puissance tend au despotisme ${ }^{39}$. Comme si la dépravation de la société s'était amorcée nécessairement par un dépassement du seuil d'altération, le mal spécifique de l'état politique se produit fatalement par une perversion de son propre élément, à savoir par la corruption du pouvoir politique qui aboutit au despotisme.

En tout état de cause, l'inégalité naturelle entre les forts et les faibles dans l'état social s'est transformée en l'inégalité artificielle entre les souverains et les sujets dans l'état politique, laquelle pourrait facilement se convertir en inégalité entre les oppresseurs et les opprimés. L'inégalité demeure, non seulement parce que le gouvernement n'arrive pas à établir l'égalité artificielle contre l'inégalité naturelle, mais aussi parce que cette mesure égalitaire induit paradoxalement une contradiction productrice de l'inégalité artificielle. Or, selon le diagnostic de Diderot sur l'État despotique de son temps, une telle contradiction de la société et du gouvernement risque de s'aggraver au point que la société rentre dans « un état de guerre; guerre du souverain contre ses sujets; guerre des sujets les uns contre les autres ${ }^{40}$.

Telle est l'histoire générale de l'homme civilisé que Diderot esquisse d'un seul jet à partir de l'origine et jusqu'au comble de l'inégalité. Mais ce n'est pas du tout une impasse, ni le dernier terme du parcours ; c'est plutôt

36. HDI, t. IV, liv. 18, ch. 42, p. 392-393 ; Mél, p. 149-150.

37. Hobbes, op. cit., VI, p. 170-171. Diderot a déjà remarqué l'inconvénient concernant la «personne physique despotique » qui occupe la place de l' «être moral souverain » dans l'article « Citoyen », Encyclopédie, DPV, t. VI, p. 467.

38. HDI, t. IV, liv. 19, ch. 2, p. 471 ; PD, p. 73.

39. HDI, t. IV, liv. 18, ch. 42, p. 394 ; Mél, p. 152. Ce jugement générique de Diderot semble plus pessimiste que ne l'est Montesquieu, cf. De l'esprit des lois, op. cit., t. I, liv. 8, ch. 10, p. 129-130 : «Les autres gouvernements périssent, parce que des accidents particuliers en violent le principe : celui-ci [le gouvernement despotique] périt par son vice intérieur ».

40. Observ, § 69, p. 543. 
un point critique qui exige une politique rénovatrice pour supprimer provisoirement ladite contradiction d'inégalité artificielle, ou bien une réouverture de la civilisation:

«Point d'individu qui, mécontent de la forme du gouvernement de son pays, n'en puisse aller chercher ailleurs une meilleure, point de société qui n'ait à changer la sienne, la même liberté qu'eurent ses ancêtres à l'adopter. Sur ce point, les sociétés en sont comme au premier moment de leur civilisation ${ }^{41}$.

S'il est frappant de voir ici l'emploi du néologisme «civilisation» (introduit en 1756 par le marquis de Mirabeau dans l'Ami des hommes ${ }^{42}$ ) pour désigner ce qui est susceptible de recommencer par le changement de gouvernement, il convient, pour l'heure, de jeter un regard sur la manière dont Diderot conçoit la temporalité de la révolution. Qu'il fasse de la révolution une occasion de revenir au moment où une société se procure comme pour la première fois un gouvernement nous rappelle sans doute un mouvement circulaire. Il s'agit du sens classique du mot « révolution », qui a sa source dans le fameux ouvrage de Copernic, De Revolutionibus orbium caelestium (1543), et dont on trouve un exemple d'application politique dans l'œuvre posthume de Hobbes parue en 1681, le Béhémoth: "J'ai vu cette révolution comme un mouvement circulaire ${ }^{43}$ - Hobbes décrit par-là l'historique de la guerre civile entre 1640 et 1660, depuis la chute de la monarchie de Stuart, par le biais de la dictature de Cromwell, jusqu'à la Restauration anglaise.

S'il est vrai que c'est dans le sillage de ce schème cyclique que Diderot parle de la révolution, encore faut-il remarquer deux choses qui nous semblent mettre en relief une spécificité de sa conception. Primo, le temps de la révolution est à la fois objectif et subjectif. Certes, aucune contrée

41. HDI, t. IV, liv. 18, ch. 42, p. 393-394 ; Mél, p. 151 (souligné par nous). Dans Le règne de la critique, dont la dernière partie est consacrée à Diderot/Raynal, Koselleck a disséqué cette dialectique du dualisme de la société et de l'État, qui mène à la crise politique : la révolution peut alors apparaître comme le jugement dernier rendu par la société moralement juste (voir op. cit., notamment p. 142-153). Malgré l'utilité de son travail, il me paraît que l'historien allemand a laissé passer la place importante accordée par Diderot à la notion d'inégalité irrémédiable, qui exige une décision provisoire, mais qui semble refuser toute solution utopique. Notons également que c'est en axant la révolution sur le processus dialectique de l'inégalité que Diderot, en tant que matérialiste athée, se démarque de Paine qui fonde la révolution sur l'égalité naturelle accordée par Dieu (cf. Edoardo Tortarolo, «La Révolution américaine dans l'Histoire des deux Indes », in L'Histoire des deux Indes : réécriture et polygraphie, Hans-Jügen Lüsebrink et Anthony Strugnell (éds.), Oxford, Voltaire Foundation, 1995, p. 217-218).

42. Cf. Catherine Larrère, «Mirabeau et les physiocrates: l'origine agrarienne de la civilisation », in Les équivoques de la civilisation, B. Binoche (éd.), Seyssel, Champ Vallon, 2005, p. 83-105.

43. Texte cité par Reinhart Koselleck, « Critères historique du concept de "révolution" des temps modernes », in Le futur passé. Contribution à la sémantique des temps historiques, trad. Jochen Hoock et Marie-Claire Hoock, Éditions de l'École des hautes études en sciences sociales, 1990, p. 66. 
n'échappe à « un cercle réglé » de prospérité et de décadence, ni à « la loi de la nature, qui veut que toutes les sociétés gravitent vers le despotisme \& la dissolution, que les empires naissent $\&$ meurent ${ }^{44}$; mais le bon moment pour la (ré)génération d'une société est indéterminé et laissé à décision de ceux qui y sont engagés, dans la mesure où leur acte de dissoudre un gouvernement est à tout moment justifiable: "Nulle autorité politique qui créée hier ou il y a mille ans, ne puisse être abrogée dans dix ans ou demain. [...] Toute autorité dans ce monde, a commencé ou par le consentement des sujets, ou par la force du maître. Dans l'un et l'autre cas elle peut finir légitimement ${ }^{45}$. Secundo, en ce qui concerne au moins la Révolution américaine, elle n'est pas du tout le retour au point de départ qui se condamne à répéter exactement le même parcours. Elle est en revanche une révolution qui fait époque, parce qu'elle comporte l'enjeu historique et mondial de créer à nouveau « la plus belle constitution qu'il y ait jamais eue parmi les hommes ${ }^{46}$ et que l'on a considérée longtemps comme irréalisable depuis Aristote jusqu'à Montesquieu : c'est-à-dire une république réalisée dans un vaste territoire comme l'Amérique septentrionale; en termes plus précis, il s'agit d'une "constitution fédérative qui ajoutait aux avantage intérieurs du gouvernement républicain » (tels que le système représentatif par lequel chaque province autonome s'oblige à "écouter tous les citoyens ») «toute la force extérieure de la monarchie » (laquelle revient au congrès général, destiné à ne s'occuper que de la diplomatie et de la défense commune $)^{47}$. Somme toute, dans la révolution telle que la conçoit Diderot se déploie une double temporalité, à savoir le cycle de la dissolutionrégénération et l'apparition du nouveau ${ }^{48}$.

\section{Les voies de la civilisation ou de la souveraineté nationale}

D'après la citation que nous avons proposée plus haut, le changement de gouvernement est un renouveau de la civilisation qui ne peut plus supporter

44. HDI, t. IV, liv. 19, ch. 2, p. 473 ; PD, p. 76.

45. HDI, t. IV, liv. 18, ch. 42, p. 394 ; Mél, p. 151-152.

46. HDI, t. IV, liv. 18, ch. 44, p. 416 ; Mél, p. 178.

47. HDI, t. IV, liv. 18, ch. 45, p. 419-420 ; Mél, p. 182. Cf. aussi HDI, t. IV, liv. 18, ch. 44, p. 412 ; Mél, p. 173 : «Un jour a fait naître une révolution. Un jour nous a transporté dans un siècle nouveau ».

48. Mutatis mutandis, on peut trouver un tel côtoiement des deux historicités dans certains penseurs des Lumières (cf. Jean-Marie Goulemot, Le règne de l'histoire. Discours historiques et révolutions XVII $-X V I I^{e}$ siècle, Paris, Albin Michel, 1996, ch. 9 : « De l'histoire cyclique à l'histoire cumulative : l'Angleterre comme lieu du débat et des compromis »). Par ailleurs, comme on a remarqué à plusieurs reprises, c'est à travers les révolutions réelles qui ont éclaté des deux côtés de l'Atlantique à la fin du XVIII ${ }^{\mathrm{e}}$ siècle que l'idée de révolution s'est évadée du schème de retour cyclique : cf. Hannah Arendt, On Revolution, London, Penguin Books, 2006, p. 37 ; Georges Gusdorf, Les principes de la pensée au siècle des Lumières, Paris, Payot, 1971, p. 423. 
l'accroissement contradictoire de l'inégalité. Il est néanmoins à noter qu'il n'y a pas une unique solution appropriée au temps de la civilisation chez Diderot. En effet, il a fourni des réponses différentes en fonction des circonstances propres à chaque nation, telles que la France, la Russie, et la colonie anglaise de l'Amérique du Nord, qui étaient les trois objets privilégiés de son discours politique dans les années 1770. Dès lors, comment peut-on comprendre cette diversification de la civilisation ainsi que son issue révolutionnaire qui n'est qu'une issue parmi d'autres ?

À ce propos, il faut s'arrêter un instant sur le concept de civilisation ${ }^{49}$ axé plus ou moins autour du contexte spécifique de sa relation avec la Russie où Diderot a trouvé un domaine d'application pour une certaine idée de développement social empruntée à l'École écossaise. L'influence du fameux essai De l'origine et du progrès des arts et des sciences (1742) de Hume est ici apparente. Pour Hume, c'est en instituant un État gouverné par la loi qui assure la personne et la propriété des citoyens qu'on est en mesure de favoriser la production économique et culturelle; si bien que la «monarchie barbare » de Pierre le Grand était tout à fait intenable dans la mesure où ce dernier, tout en voulant imiter le raffinement des Européens, n'en avait pas moins tenu le peuple russe en sujétion ${ }^{50}$. Voilà pourquoi, comme on peut le lire dans les Mélanges philosophiques, historiques, etc., pour Catherine II, Diderot exige que l'Impératrice ne tombe jamais dans les mêmes errements que le précédent Tsar, et qu'elle se lance au contraire dans la mise en place de préliminaires destinés à civiliser le peuple russe encore barbare. Il s'agit de commencer coûte que coûte par l'affranchissement des serfs malmenés afin d'en former un tiers état qui constituerait non seulement un noyau de la nation jouissant pleinement d'une égale liberté juridique, mais aussi un noyau de population productrice d'arts autochtones ${ }^{51}$. À cette nouvelle classe de citoyens, il faut encore accorder, en sus d'un droit à la jouissance paisible, le «droit d'opposition» ou la liberté au sens politique, au moyen surtout de la mise en place d'une commission ou d'une assemblée législative

49. Sur ce concept, il existe de nombreuses études parmi lesquelles nous consultons : Georges Dulac, «Diderot et "la civilisation" de la Russie », in Denis Diderot 1713-1784, Colloque international, Paris-Sèvres-Reims-Langres (4-11 juillet 1984), Anne-Marie Chouillet (éd.), Paris, Aux amateurs de livres, 1985, p. 161-171; Id., «Quelques exemples de transferts européens du concept de "civilisation", 1765-1780», in Les équivoques de la civilisation, op. cit., p. 106-135 ; Gianluigi Goggi, «Diderot et l'abbé Baudeau : les colonies de Saratov et la civilisation de la Russie », Recherches sur Diderot et sur l'Encyclopédie, $\mathrm{n}^{\circ} 14,1993$, p. 23-83; Id., «Diderot et le concept de civilisation », Dix-huitième siècle, $\mathrm{n}^{\circ} 29,1997$, p. 353-373.

50. David Hume, De l'origine et du progrès des arts et des sciences, in Essais et traités sur plusieurs sujets. Essais moraux, politiques et littéraires, trad. Michel Malherbe, Paris, Vrin, 1999, t. 1, p. 171. Dans la même lignée, Adam Ferguson insiste aussi sur l'indivisibilité de la liberté politique et du développement économique (An Essay on the history of civil society, Fania Oz-Salzberger (éd.), Cambridge, Cambridge University Press, 1995, p. 139).

51. MC2, VII, p. 239 ; XI, p. 243. Sans utiliser le mot «civilisation », Diderot a déjà présenté la même idée dans Fragments politiques échappés du portefeuille d'un philosophe, op. cit., p. $172-175$. 
représentante de la nation entière ${ }^{52}$. Civiliser la Russie, c'est donc transformer l'État rude et autocratique en État libre et florissant, en créant parallèlement le sujet collectif pourvu de «l'autorité populaire»; ce qui impliquerait, si l'on allait jusqu'au bout, la suppression du despotisme par le despote lui-même.

Cependant, l'initiative réformatrice d'un souverain, quand même on pourrait s'y attendre, n'est pas capable de tout créer dans le vide. Elle doit plutôt agir sous la contrainte de poids historiques qui pèsent sur une situation. Il s'agit de savoir si telle ou telle nation est en état de se libérer ou en voie de se corrompre, autrement dit, en quel sens elle est, ou n'est pas, civilisée. Sur ce point, il est certain que Diderot fait référence à la quatrième observation de l'Essai en question de Hume, qui constate la décadence quasi inévitable d'une nation une fois parvenue à la perfection des arts et des sciences $^{53}$; mais, s'agissant de la possibilité d'une émancipation, il se réfère davantage au chapitre 8 du livre II du Contrat social, où Rousseau fait la distinction entre le peuple dans sa jeunesse qui «peut acquérir la liberté » et celui dans sa vieillesse «qui ne la recouvre jamais ${ }^{54}$. S'inscrivant dans cette lignée, Diderot distingue aussi dans les Observations sur le Nakaz « un peuple policé » et «un peuple à policer »: «la condition de celui-là me paraît pire que la condition de celui-ci ; l'un est sain et l'autre est attaqué d'un vieux mal presque incurable ${ }^{55}$. Ainsi, en ce qui concerne la Russie et la France, bien que leurs gouvernements fussent également autocratiques, il en a tiré deux conclusions opposées ${ }^{56}$. On peut attendre de la nation russe qu'elle soit un «homme vigoureux et sauvage qui naît»; et c'est dans de telles conditions que la politique civilisatrice de Catherine II peut s'effectuer. A contrario, il était trop tard pour qu'un monarque français pût rétablir sa nation, puisqu'elle était comparable à « un homme délicat et maniéré attaqué d'une maladie presque incurable », en ayant perdu par le coup d'État de Maupeou en 1771 le Parlement qui avait été le seul frein contre le pouvoir arbitraire $^{57}$. Cela n'exclut pourtant pas que la France corrompue par l'Ancien Régime puisse renaître en suivant une autre voie qu'une réforme par en haut. Contrairement à Rousseau pour qui la régénération-libération ne survient qu'une fois chez un peuple barbare (Sparte au temps de Lycurgue par exemple), celle d'un peuple policé est assez concevable pour Diderot ; à preuve la façon qu'il a de parler métaphoriquement d'un rajeunissement par

52. MC2, XXIV, p. 274 et suiv.

53. Hume, op. cit., p. 187.

54. Du contrat social, in Euvres complètes, op. cit., t. III, p. 385.

55. Observ, § 19, p. 521.

56. Sur cette comparaison, voir Bertrand Binoche, La raison sans l'Histoire, Paris, Presses Universitaires de France, 2007, ch. 3 : «Diderot et Catherine II, ou les deux histoires ».

57. MC2, XI, p. 245. Au sujet de l'incurabilité de la monarchie française après le coup d'État de Maupeou, cf. MC2, I, p. 250 : «En conséquence, l'ordre public ou notre gouvernement a été si parfaitement détruit que je ne pense pas que la toute-puissance et l'infinie bonté du roi, qui n'y pense sûrement pas, pût le rétablir. La Confiance est perdue ». 
le renversement de la monarchie française dans la Réfutation d'Helvétius : «Comme Médée rendit la jeunesse à son père ; en le dépeçant et le faisant bouillir $»^{58}$.

Cela dit, dans le cas du Nouveau Monde, alors que le peuple naissant de la colonie de l'Amérique du Nord était loin de se corrompre, il a souffert de l'empire de l'Angleterre qui pouvait «disposer à son gré de son gouvernement, de ses lois, de son commerce ; l'imposer comme il lui plaît ; limiter son industrie et l'enchaîner par des prohibitions arbitraires $»^{59}$. À cet égard, il était empêché de s'éveiller à la civilisation, assujetti à un règne despotique où la contradiction de l'inégalité artificielle s'aggravait, comme dans les deux grands pays européens de l'Ancien Monde. Il y a toutefois une particularité propre au despotisme colonial: ici, la domination absolue s'exerce moins par un tyran contre ses sujets que par une mère patrie contre sa colonie, d'où vient que le peuple de la colonie ne peut espérer son émancipation ni par la réforme d'un souverain, ni par le tyrannicide :

«On ne peut attendre la liberté que d'une rupture, dont la suite est la ruine de l'une ou l'autre nation, et quelquefois de toutes les deux. Le tyran est un monstre à une seule tête, qu'on peut abattre d'un seul coup. La nation despote est une hydre à mille têtes qui ne peuvent être coupées que par mille glaives levés à la fois ${ }^{60}$.

C'est ainsi que la Révolution américaine a trouvé son issue sous la forme de l'indépendance: la colonie y est entrée en guerre avec sa mère patrie (la nation despote) pour s'en séparer comme une nation autonome. Et si cette "démarche qui rompait des nœuds formés par le sang, par la religion, \& par l'habitude, devait être soutenue par un grand concert de volontés ${ }^{61}$, elle équivaut à la formation d'une société naturelle qui tire son origine d'une convergence des vues de ses membres pour lesquels le pouvoir gouvernemental doit exister. Cette rupture coloniale est donc un moment de l'auto-organisation soudaine d'une nouvelle nation qui se réalise elle-même en souveraineté populaire, contrairement au peuple russe qui nécessiterait,

58. Réfutation d'Helvétius, DPV, t. XXIV, p. 483. Diderot qualifie cette régénération d'un peuple corrompu de «l'ouvrage d'une longue suite de révolutions » (HDI, t. III, liv. 11, ch. 4, p. 102-103; PD, p. 71). C'est ainsi que Goggi insiste sur l'importance de la première révolution anglaise chez Diderot comme référence historique d'une régénération éventuelle des Européens, tout en délimitant la portée de la Révolution américaine comme cas singulier (De l'Encyclopédie à l'éloquence républicaine, op. cit., p. 528-536). Mais cette portée ne s'élargirait-elle pas si nous dirigeons nos yeux hors d'Europe où la libération d'indépendance est en jeu ? En effet, Diderot affirme l'impact global de la Révolution américaine : «Nous allons, dans ce moment, décider du sort d'une race d'hommes plus nombreuse peut-être que tous les peuples de l'Europe ensemble. [...] Imaginons-nous que toutes les générations du monde à venir ont dans ce moment les yeux fixés sur nous, \& nous demandent la liberté » (HDI, t. IV, liv. 18, ch. 44, p. 416 ; Mél, p. 178).

59. HDI, t. IV, liv. 18, ch. 42, p. 395 ; Mél, p. 153.

60. HDI, t. IV, liv. 18, ch. 42, p. 395-396 ; Mél, p. 153-154 (souligné par nous).

61. HDI, t. IV, liv. 18, ch. 45, p. 419 ; Mél, p. 182 (souligné par nous). 
pour se civiliser et obtenir sa souveraineté, la médiation indispensable de l'Impératrice. De là, on peut conclure que la différence de voies de la civilisation est celle de modes d'institution de la souveraineté nationale, soit par la révolution, soit par la réforme.

L'adhésion de Diderot à la souveraineté nationale décèle à travers ses écrits sur la Russie jusqu'à l'Histoire des deux Indes son penchant démocratique en contradiction avec la tendance absolutiste de Hobbes. Pour ce dernier, toute décision politique, à commencer par la législation, relève exclusivement d'une personne souveraine qui s'en empare par la substitution de son unique volonté à celle des contractants, de sorte qu'elle peut exercer le pouvoir arbitraire à la soumission auquel aucun sujet n'est soustrait puisqu'il est présumé y consentir. Tandis que cette unification des volontés par l'aliénation totale sert de base à l'État hobbesien comme union artificielle, la société diderotienne, en s'instituant comme État, se réfère toujours au principe d'association naturelle qui prend de la consistance dans une convergence spontanée des volontés inaliénables. Les individus qui composent une nation ne peuvent donc s'obliger qu'à leur propre législation, pour que le pouvoir étatique s'exerce au profit d'eux-mêmes :

«Il n'y a point de vrai souverain que la nation; il ne peut y avoir d'autre vrai législateur; il est rare qu'un peuple se soumette sincèrement à des lois qu'on lui impose ; il les aimera, il les respectera, il y obéira, il les défendra comme son ouvrage, s'il en est lui-même l'auteur. Ce ne sont plus les volontés arbitraires d'un seul, ce sont celles d'un nombre d'hommes qui ont consulté entre eux sur leur bonheur et leur sécurité $»^{62}$.

Bien entendu, Diderot se rend compte d'une brèche de facto éventuelle au sein de ce «nous", nation de jure indivisible, à savoir d'une contradiction entre l'exécutif et la législation; et c'est pourquoi il lui importe de codifier le droit de destitution ou de résistance du peuple pour «qu'il nous poursuive, qu'il nous dépose et même qu'il nous condamne à mort si le cas l'exige [= en tant que nous serions un souverain illicite] $\gg^{63}$. Mais une telle codification de la souveraineté nationale suffit-elle pour la pérenniser? Si le peuple de l'Amérique septentrionale a acquis à travers la révolution d'indépendance son propre gouvernement représentatif, n'aurait-il pas à craindre que les députés n'agissent à l'encontre de ses volontés ? Après avoir mentionné des avantages de la constitution fédérative des États-Unis, Diderot ajoute en réalité : si le peuple n'a pas encore foi en sa constitution, « il faut que tous les citoyens soient sans cesse au conseil, à l'armée, dans la place publique, \& qu'ils aient les yeux toujours ouverts sur les représentants à qui ils ont confié leur destinée ${ }^{64}$. Le peuple doit être sans cesse prêt à

62. Observ, § 1, p. 508. Voir MC2, XXIV, p. 277 : «C'est le concours et l'opposition des volontés générales aux volontés particulières, l'avantage spécial de la démocratie sur toutes les autres espèces de gouvernement ».

63. Observ, § 1, p. 508.

64. HDI, t. IV, liv. 18, ch. 45, p. 421 ; Mél, p. 183-184. 
recourir au coup de force pour entretenir ou rétablir l'intégralité de son statut double comme nation-souverain, au cas où le lien entre les représentants et les représentés risque de se dénouer. De même que l'inégalité originelle parmi les hommes qui s'accumule contradictoirement par sa solution même (le gouvernement) les a contraints à l'issue révolutionnaire, de même la précarité immanente de l'institution d'une souveraineté nationale exige en cas de nécessité une solution extérieure à elle-même. Ainsi faut-il à nouveau limiter l'institution et laisser de la marge à l'action politique du côté du peuple.

\section{Le droit et la passion de la révolution : radicaliser Locke}

Il faut maintenant revenir sur notre question initiale à laquelle nous pouvons répondre en partie comme suit: la Révolution américaine est justifiable pour autant qu'elle est une application circonstancielle de la phase prospère de la civilisation à laquelle sont corrélés l'historique (la sortie de la barbarie ou de la corruption du civilisé), le politique (l'incarnation de l'État libre sous la forme de la souveraineté nationale) et l'économique (le développement du commerce et de l'industrie qui sert de base au progrès des sciences et des arts). Si cela nous permet de mesurer la distance que Diderot entretient par rapport à l'absolutisme contractualiste de Hobbes, en est-il ainsi de sa distance par rapport à Locke, qui a consacré le dernier chapitre du second Traité du gouvernement à la dissolution du gouvernement et au droit de résistance ? Il convient pour le savoir de comparer à présent Diderot avec Locke, d'autant plus que le premier radicalise certaines thèses du second pour théoriser la logique interne du mouvement révolutionnaire. Dans cette perspective, nous distinguerons deux aspects différents : la légitimation du droit de révolution et l'explication génétique ou psychologique de celle-ci.

À l'évidence, lorsque Diderot compare la colonie de l'Amérique du Nord aux «enfants » et l'Angleterre aux «parents», et lorsqu'il justifie la totale liberté qu'a celle-là de changer le gouvernement créé par celle-ci, il s'appuie sur une comparaison lockéenne avec le « fils » (Son) et le «père » (Father). À l'encontre de Filmer qui défend le pouvoir patriarcal du monarque civil, Locke affirme que les fils sont «libres de se séparer de leurs familles et du gouvernement ${ }^{65}$ pour les former à nouveau, parce que «on ne saurait obliger ses enfants ou sa postérité par un contrat, quel qu'il soit. Car le fils, une fois devenu adulte, est exactement aussi libre que son père, de sorte que, par l'un de ses actes, le père ne peut pas plus aliéner la liberté de son fils que celle d'aucun autre homme ${ }^{66}$. Pareillement, Diderot insiste sur la liberté de la génération présente qui ne se charge d'aucune obligation par rapport à la génération passée, et qui peut délégitimer à

65. Locke, op. cit., $\S 115$, p. 84-85.

66. Ibid., § 116, p. 85. 
son gré le gouvernement existant, quoiqu'il ait été formé légitimement par l'accord de celle-ci : «Le consentement des aïeux ne peut obliger les descendants ; et il n'y a point de condition qui ne soit exclusive du sacrifice de la liberté ${ }^{67}$. Quoique Diderot ait une dette envers Locke, il le surpasse en ce qu'il donne à cette liberté de changer le gouvernement le titre de droit naturel:

«Si les peuples sont heureux sous la forme de leur gouvernement, ils la garderont. S'ils sont malheureux, ce ne seront ni vos opinions, ni les miennes; ce sera l'impossibilité de souffrir davantage et plus longtemps qui les déterminera à la changer, mouvement salutaire que l'oppresseur appellera révolte, bien qu'il ne soit que l'exercice légitime d'un droit inaliénable et naturel de l'homme qu'on opprime, et même de l'homme qu'on n'opprime pas ${ }^{68}$.

Bref, tant que la révolution est un droit inaliénable et naturel de l'homme, tout le monde peut l'exercer selon sa propre volonté, sans condition. En ce sens, Diderot supprime les conditions posées par Locke à l'exercice du droit de résistance qui vise à dissoudre le gouvernement. Selon le dernier, la résistance est justifiable dans le cas où le gouvernement viole le droit ou la propriété du peuple de telle manière qu'il agit à l'encontre de la mission (Trust) pour laquelle il a été constitué ; et c'est du jugement du peuple que dépend la conformité du gouvernement à sa mission ou sa légalité ${ }^{69}$. Diderot reprend assurément ce statut du peuple comme juge ultime, mais il ne lui importe pas de définir un critère plus ou moins objectif et légal de ce qui autorise le peuple à juger le bien-fondé de son gouvernement $^{70}$; car, même si le peuple ne gémit pas sous l'oppression, sa volonté de rompre le statu quo pour améliorer sa situation est justifiable en soi. À la limite, s'il y a un critère, c'est le bonheur tel que le peuple le conçoit tout subjectivement. D'ailleurs, Locke semble établir une distinction substantielle entre la résistance juste qui remplit ladite condition justifiante et la rébellion illicite qui tient tête par force à l'autorité légale, que cette rébellion soit le fait du gouvernant ou du sujet ${ }^{71}$. À cet égard, Diderot n'en admet que la distinction nominale; en effet, si toute tentative de révolution

67. HDI, t. IV, liv. 18, ch. 42, p. 396 ; Mél, p. 154.

68. HDI, t. IV, liv. 18, ch. 42, p. 393 ; Mél, p. 151 (souligné par nous).

69. Locke, op. cit., § 221, p. 158 et suiv; $§ 240$, p. 174.

70. En revendiquant le droit de résistance à l'instar de Locke, d'Holbach exige que l'injustice des princes soit objectivement jugée à la lumière d'un critère de légalité qu'il appelle «Loix [sic] fondamentales », dans le souci d'éviter «le caprice et le ressentiment du citoyen aveugle» (Système social, Josiane Boulad-Ayoub (éd.), Paris, Fayard, 1994, II, ch. 5, p. 285). Pour l'analyse minutieuse de la distance entre d'Holbach et Diderot autour de l'auteur du Traité du gouvernement, cf. Goggi, op. cit., p. 408-421.

71. Locke, op. cit., §226, p. 162-163: «Car la rébellion n’est pas une opposition aux personnes mais à l'autorité, laquelle ne peut être fondée que sur la constitution et les lois du gouvernement. Par suite, quiconque enfreint celles-ci et tente de faire prévaloir cette violation par la force, celui-là est proprement rebelle ». 
est justifiable, il ne reste plus que des différences d'appellation par rapport à un seul et même événement, soit la rébellion injuste aux yeux des gouvernants, soit l'exercice légitime d'un droit de la part du peuple. De surcroît, c'est encore à travers la lutte autour d'un nom que la Révolution américaine considérée d'abord comme non-droit en arrive à se rendre justice : "commençons par déclarer notre INDÉPENDANCE [sic]. Elle seule peut effacer le titre de sujets rebelles que nos insolents oppresseurs osent nous donner $»^{72}$.

Malgré cela, l'exercice du droit naturel et inaliénable à changer le gouvernement ne serait-il pas contraint à l'oppression d'autant qu'il est assimilé à une sédition intolérable du point de vue des gouvernants ? Il est aisé de voir que l'on ne peut exercer le droit de révolution qu'en risquant la mort. Aussi Hobbes déclare-t-il dans Du Citoyen que si les individus n'en viennent pas à avoir un «espoir de vaincre » en supputant les chances de réussite de l'insurrection, ils "dissimuleront et préféreront supporter une charge lourde plutôt qu'une autre plus lourde encore ${ }^{73}$, c'est-à-dire une défaite périlleuse. Par ailleurs, chez Locke, alors même que le peuple «est plus disposé à souffrir qu'à rétablir ses droits par la résistance ${ }^{74}$, il cessera de supporter ses gouvernants aussitôt qu'une longue suite d'injustices révélera indéniablement leurs malveillances, leurs infractions continuelles à leur mission. Diderot indique aussi cette sorte de seuil de tolérance dont le dépassement susciterait probablement une insurrection populaire, mais ce n'est pas tant une transgression de la mission qu'un sentiment des infortunés: "Sous quelque gouvernement que ce soit, la nature a posé des limites au malheur des peuples. Au-delà de ces limites, c'est ou la mort ou la fuite ou la révolte ${ }^{75}$; "Il n'y a jamais que le malheureux ou l'opprimé qui se révolte ${ }^{76}$. Si la révolte apparaît de fait toujours comme une réaction contre l'oppression dans un extrême malheur, c'est que tout exercice du droit à l'insurrection accompagne inévitablement le sacrifice d'une partie du peuple à travers la guerre contre le gouvernement. La légitimité du droit de révolte-révolution ne sert donc pas d'amorce à une genèse effective de celle-ci.

C'est ainsi que, en se demandant pourquoi le peuple se révolte rarement contre l'oppression, Diderot est amené à chercher au-delà de Locke une condition empirique requise pour la révolte du côté de la psychologie des engagés :

72. HDI, t. IV, liv. 18, ch. 44, p. 416 ; Mél, p. 179. C'est ce que Paolo Quintili appelle « un "paradoxe de la politique" : le droit s'inaugure et se présente, dans l'histoire, par le moyen du non-droit» - paradoxe que Diderot partage avec Kant («Éthique universaliste, politique révolutionnaire. Un rapport, un problème ouvert. Diderot, Raynal, Kant », in L'idée de révolution: quelle place lui faire au XXI siècle?, Olivier Bloch (dir.), Paris, Publications de la Sorbonne, 2009, p. 84).

73. Hobbes, op. cit., XII, p. 250.

74. Locke, op. cit., § 230, p. 165.

75. Réfutation d'Helvétius, DPV, t. XXIV, p. 483.

76. Observ, § 44, p. 530. 
«C'est qu'une société est un assemblage d'hommes occupés de différentes fonctions, divisés d'intérêt, jaloux, pusillanimes, préférant la jouissance paisible de ce qu'on leur laisse à la défense armée de ce qu'on leur enlève, vivant à côté les uns des autres, se pressant, sans aucun concours de volontés : c'est que ce concert, si raisonnable, si utile, quand il subsisterait entre eux, ne leur donnerait ni le courage, ni la force qui leur manque, ni par conséquent ou l'espoir de vaincre, ou la résolution de périr : c'est qu'ils ne verraient dans le succès que l'avantage de leur descendants, qu'il aiment moins qu'eux.... Cependant il est arrivé quelquefois.... Oui, par l'enthousiasme du fanatisme.... ${ }^{77}$.

Certes le concours de volontés est ici comme là une condition nécessaire en tant qu'il organise plusieurs personnes en sujets politiques tendus vers un but commun, mais ce principe de collectivité n'est pas une condition suffisante. Pour qu'une révolte-révolution advienne effectivement, il faut encore une autre condition essentielle, à savoir l'enthousiasme du fanatisme, principe affectif qui presse les insurgés de défier bravement le régime en place au mépris de tout danger. Que Diderot voie dans la Révolution américaine un moment privilégié où lesdites conditions sont remplies, cela est évident du fait qu'il exhorte les «Prêtres du Nouveau Monde» à « promettre le salut éternel à ceux qui périront les armes à la main martyrs de la liberté » et à louer « un fanatisme plus heureux, né de la politique et de la liberté $»^{78}$.

Notons en guise de conclusion que c'est en renversant une thèse de Hobbes concernant le danger politique de l'émotion que Diderot finit par remplir la tâche de justifier la révolution dans la temporalité de la civilisation, c'est-à-dire de déterminer les conditions de facto de la révolution de jure. Comme sa préoccupation majeure est la paix par l'État, Hobbes se défie de tout ce qui incite les passions à la rébellion comme la doctrine fanatique qui s'oppose à l'obéissance civile ou l'éloquence qui excite le peuple à l'insurrection ${ }^{79}$. Sans doute Hobbes se persuade-t-il que si l'homme agit rationnellement en tenant compte de son propre intérêt au lieu de se laisser aller à un mouvement tumultueux de passions, il n'osera pas délaisser sa situation actuelle ni se vouer à la lutte périlleuse pour en chercher témérairement une meilleure. En revanche, pour Diderot, un tel dévouement sacrificiel né de la passion politique orientée vers le changement fait précisément l'objet de justifications. S'il est vrai qu'il peut défendre la révolution parce qu'il ne vit plus à une époque où la guerre civile et religieuse était le péril majeur, il est pourtant loin d'être optimiste. En effet, la révolution ne renouvelle que le processus instable de la civilisation d'une nation, lequel n'a rien à voir avec le progrès indéfini ni avec

77. HDI, t. IV, liv. 19, ch. 6, p. 594 ; PD, p. 210-211 (souligné par nous).

78. HDI, t. IV, liv. 18, ch. 42, p. 402-403 ; Mél, p. 162.

79. Hobbes, op. cit., XII, § 12. Sur le renversement diderotien de Hobbes à propos de la fonction politique de l'éloquence républicaine affective, cf. Goggi, op. cit., p. 473-477; p. 574-575. 
l'équilibre perpétuel. Et même si l'on en arrive à constituer une démocratie, «malheureusement, cet état de bonheur n'est que momentané » ${ }^{80}$, sans excepter les États-Unis qui, comme on l'a vu, ne se soustraient pas à la corruption. Seulement, en tant que philosophe de l'époque où la colonisation du monde par des Européens est bousculée par l'indépendance coloniale, Diderot semble concevoir la nécessité pour une nation tenue en sujétion par une autre de s'en émanciper de manière à disposer de son autonomie. Une civilisation dont l'acte inaugural est la ressaisie par le peuple de sa liberté politique s'annonce du moins sous de bons auspices, malgré le caractère toujours aléatoire de son avenir.

80. HDI, t. IV, liv. 19, ch. 2, p. 473 ; PD, p. 76. C'est pourquoi Diderot attend au plus des Américains ceci : «Puissent-ils reculer, au moins pour quelques siècles, le décret prononcé contre toutes les choses de ce monde; décret qui les a condamnées d'avoir leur naissance, leur temps de vigueur, leur décrépitude et leur fin » (Essai sur les règnes de Claude et de Néron, DPV, t. XXIV, p. 355). 